\title{
Gas cells for tunable diode laser absorption spectroscopy employing optical diffusers. Part 1: Single and dual pass cells.
}

\author{
J Hodgkinson $^{1}$, D Masiyano ${ }^{1,2}$ and R P Tatam ${ }^{1}$ \\ 1 Engineering Photonics Group, School of Engineering, Cranfield University, Bedfordshire, \\ MK43 0AL, UK. \\ 2 Now at: Alps Electric (UK) Ltd, Garamonde Drive, Wymbush, Milton Keynes, MK8 8LW, UK. \\ * corresponding author j.hodgkinson@cranfield.ac.uk
}

\begin{abstract}
New designs for gas cells are presented that incorporate transmissive or reflective optical diffusers. These components offer simple alignment and can disrupt the formation of optical etalons. We analyse the performance-limiting effects in these cells of random laser speckle (both objective and subjective speckle), interferometric speckle and self-mixing interference, and show how designs can be optimised. A simple, single pass transmissive gas cell has been studied using wavelength modulation spectroscopy to measure methane at $1651 \mathrm{~nm}$. We have demonstrated a short-term noise equivalent absorbance (NEA, $1 \sigma)$ of $2 \times 10^{-5}$, but longer term drift of up to $3 \times 10^{-4}$ over 22 hours.
\end{abstract}

\section{PACS codes}

07.07.Df sensors - chemical

42.62.Fi laser spectroscopy

42.30.Ms Speckle and moire patterns

42.25.Fx diffraction and scattering 


\section{Introduction}

Tunable diode laser spectroscopy (TDLS) is of great interest for gas detection in a variety of applications including health and safety, industrial process control, emissions monitoring and healthcare. The laser diode emission is scanned across a single absorption line of the target gas, and / or modulated in wavelength modulation spectroscopy (WMS) ${ }^{[1]}$. Many practical implementations of these techniques have shown that the detection sensitivity is limited by interference fringes and not by the theoretical limit given by detector noise ${ }^{[2,3]}$. The interference fringes stem from Fabry-Pérot etalons between reflecting or scattering surfaces such as mirrors, lenses, optical fibre end faces, detector and laser head windows, semiconductor surfaces, and components of multipass cells ${ }^{[1]}$, and also from low levels of optical feedback to the laser diode $\mathrm{e}^{[4]}$.

The etalons often exhibit a free spectral range similar to the linewidth of the absorbing species and appear as periodic spectral features with sufficient amplitude to obscure weak absorption signals ${ }^{[5]}$. Bomse et al. ${ }^{[6]}$ suggested that gas cell windows are often the worst culprits. Narrow laser linewidths (tens of megahertz) used to resolve individual gas lines have correspondingly long coherence lengths (tens of metres). An etalon formed between the windows of a gas cell with a typical length of $10 \mathrm{~cm}$ will give rise to fringes with a spacing of approximately $1 \mathrm{GHz}\left(0.033 \mathrm{~cm}^{-1}\right)$, which is comparable to gas absorption linewidths at atmospheric pressure.

A number of techniques have been used to reduce interference fringes in TDLS, which in general fall into three broad categories. In the first, a frequency jitter is applied to the laser diode and the signal is integrated, averaging out the fringes ${ }^{[7]}$. The second category involves post detection filtering using high pass filters ${ }^{[1]}$, low pass filters ${ }^{[8]}$ or Fourier domain analysis ${ }^{[9]}$. However, neither category can deliver an improvement in signal to noise ratios for fringes whose FSR is comparable to the gas absorption linewidth ${ }^{[1]}$. In the final category, the fringe spacing is mechanically modulated and the resultant signal is integrated to average zero, or to minimise the contribution of the fringes to the absorption signal. This has been implemented using longitudinal dithering of optical elements ${ }^{[6]}$, an oscillating mirror ${ }^{[2,10]}$ and an oscillating Brewster-plate spoiler ${ }^{[11]}$. While effective, this approach limits the available detection bandwidth and adds to system complexity.

All the above approaches benefit if etalon formation is minimised, by reducing and misaligning Fresnel reflections. Standard designs for TDLS gas cells therefore include wedging and angling of all windows, 
antireflection coating of windows and lens surfaces, and angle polishing optical fibre ends. This results in tight alignment tolerances that are difficult to fabricate and to maintain in the field ${ }^{[12]}$. We present the results of a new approach using optical diffusers in reflection or transmission. These components offer simple alignment and can disrupt the formation of optical etalons within the gas cell. However, their use introduces new issues to be considered that can also limit performance, namely laser speckle and the effects of optical feedback. In Part 1 of this paper we show how an understanding of these issues, and how they relate to TDLS, can be used to optimise the design of simple, single or dual pass cells. Both objective speckle (without a lens) and subjective speckle (in which a lens is used to collect light onto the photodetector) are considered. We report the results of a comparison of different cell geometries at the design stage, and experiments with a simple transmissive geometry. In Part 2 of this paper we also report a study of the same performance-limiting effects in integrating spheres - the optical diffuser analogue of multipass cells.

Interest in this approach is growing. Chen et al. have used a diffuse reflector manufactured from anodised aluminium in an industrial oxygen sensor ${ }^{[13]}$. A group at Lund University have used TDLS to explore porous and scattering media including pharmaceutical samples ${ }^{[14]}$ and biological tissue ${ }^{[15]}$. Finally, the reflective cells that we consider here have much in common with the standard configuration for laser pointer style gas detectors, in which light from the laser is backscattered from an uncontrolled and typically diffusely reflecting background target ${ }^{[16,17,18]}$.

\section{Theory}

\subsection{Gas detection}

Methane has a well-known $2 v_{3}$ absorption band in the near infrared region of the spectrum, centred around $1.66 \mu \mathrm{m}$, shown in Figure 1. We have used the R4 quadruplet line at $1.651 \mathrm{~nm}$, however our results are generally applicable to TDLS based detection of other gases using different wavelengths of operation. 


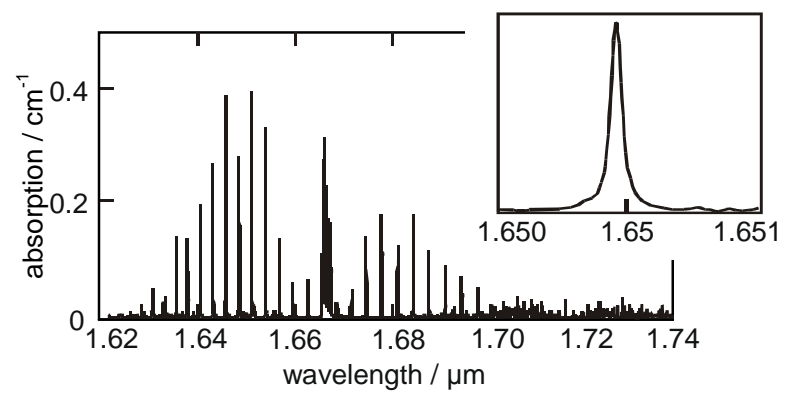

Figure 1. Absorption spectrum for $100 \%$ methane in the near infra-red, calculated using data from the HITRAN database ${ }^{[19]}$.

For monochromatic radiation, the level of light transmitted through a gas cell is given by the BeerLambert law;

$$
\frac{I}{I_{0}}=\exp (-\alpha z)
$$

where $I_{0}$ is the light power transmitted in the absence of light absorption, $\alpha$ is the absorption coefficient of the measurand in $\mathrm{cm}^{-1}$ (equal to the specific absorptivity of the gas $\varepsilon$ multiplied by the gas concentration) and $z$ is the pathlength (here in $\mathrm{cm}$ ). The gas absorptivity is often expressed in units of $\mathrm{cm}^{-1}$ per unit partial pressure of gas; at $1651 \mathrm{~nm}$ and at atmospheric pressure, methane has a value of $\varepsilon$ at the line centre of $0.38 \mathrm{~cm}^{-1} \mathrm{~atm}^{-1}$ [19]. At low values of $\alpha z$, equation (1) approximates to the following linear relationship, for which the proportional change in detected signal is given by.

$$
\frac{\Delta I}{I_{0}} \approx \alpha z
$$

We can therefore describe the uncertainty in the gas detection measurement as $\Delta \mathrm{I} / \mathrm{I}_{0}$, and translate this directly to a limit of detection for a given absorption coefficient. For wavelength modulation spectroscopy (WMS) using $2^{\text {nd }}$ harmonic demodulation, a scale factor applies based on the modulation index used (the ratio of wavelength modulation amplitude to linewidth at half width half maximum). For an absorption line with a Lorentzian profile (typical for measurements at atmospheric pressure), the $2^{\text {nd }}$ harmonic component $I_{2}$ is given by ${ }^{[20]}$

$$
\frac{I_{2}}{I_{0}}=-2 K \alpha z
$$

where the constant $K$ can be optimised to a value of 0.343 by setting the modulation index to a value of $2.2^{[21]}$. For methane at $1651 \mathrm{~nm}$ at atmospheric pressure, this corresponds to a dither of $\Delta v= \pm 2.8 \mathrm{GHz}$ or $\Delta \lambda= \pm 0.025 \mathrm{~nm}$. 


\subsection{Random laser speckle}

Laser speckle patterns are formed when coherent light is backscattered from a randomly scattering surface whose surface roughness is comparable with the wavelength of illumination. The speckle is a superposition of random interferences and can be treated by assuming that the bright speckles are independent and obey Poisson statistics ${ }^{[22]}$ such that the standard deviation of a single speckle is equal to the mean intensity. A detector placed within the speckle field integrates over a finite number of speckles; if $N$ speckles are integrated, the uncertainty in the measured intensity is therefore $1 / \sqrt{ } N$. Random speckle noise therefore depends on the speckle size $\varepsilon$. We can identify two distinct forms of speckle as shown in Figure 2, namely objective and subjective speckle.



Figure 2. Creation of objective and subjective speckle by laser illumination of a diffusely reflective surface.

For objective speckle, the speckle diameter $\varepsilon_{0}$ is given by ${ }^{[23]}$

$$
\varepsilon_{0}=1.2 \frac{\lambda z}{d}
$$

where $z$ is the distance from the optical diffuser to the detector and $d$ is the illuminated extent of the diffuser. Note that this equation assumes that $d<<z$. For subjective speckle, we have ${ }^{[23]}$

$$
\varepsilon_{s}=1.2 \frac{\lambda L}{a}
$$

where $L$ is the distance from lens to detector, and $a$ is the diameter of the lens aperture. If there are $N$ independent speckles, the RMS deviation from a zero baseline for either form of speckle is given by 


$$
\frac{\Delta I}{I_{0}}=\frac{1}{\sqrt{N}}=\frac{\varepsilon}{D}
$$

where $D$ is diameter of the detector. In the worst case, if the deviations cannot be removed (eg by baseline subtraction), they translate to uncertainty in the measurement. We have evaluated the resulting uncertainty as $\Delta I / I_{0}$ for both types of speckle, making some assumptions about the cell geometry. The results are shown in Table 1.

Table 1. Estimated uncertainties in detected signals for random laser speckle

\begin{tabular}{|l|c|c|}
\hline Type of speckle & $\begin{array}{c}\underline{\text { Estimated }} \\
\underline{\text { uncertainty in } \Delta I / I_{0}}\end{array}$ & $\begin{array}{c}\text { Estimated change in } \Delta I / I_{0} \\
\text { under wavelength modulation }\end{array}$ \\
\hline Objective speckle (no lens) $^{\mathrm{a}}$ & $8 \times 10^{-3}$ & $8 \times 10^{-3}-8 \times 10^{-7}$ \\
\hline Subjective speckle (with lens) $^{\mathrm{b}}$ & $2 \times 10^{-3}$ & $2 \times 10^{-3}-2 \times 10^{-7}$ \\
\hline
\end{tabular}

$$
\begin{array}{ll}
\text { a } & z=100 \mathrm{~mm}, d=25 \mathrm{~mm}, \lambda=1651 \mathrm{~nm}, D=1 \mathrm{~mm} \\
\text { b } & z=100 \mathrm{~mm}, a=25 \mathrm{~mm}, L=25 \mathrm{~mm}, \lambda=1651 \mathrm{~nm}, D=1 \mathrm{~mm} \\
\text { c } & \Delta \lambda=0.025 \mathrm{~nm}, h \geq \lambda
\end{array}
$$

Now we consider the effect of wavelength modulation, for in many cases it is not simply the speckle that is of concern, rather how that speckle pattern changes with wavelength. For the small wavelength scans typical of TDLS, the speckles remain essentially in place, however each speckle moves in and out of phase. Fujii and Lit have calculated the mean deviation in the measured intensity at a single on-axis point for a change in wavelength $\Delta \lambda^{[24]}$, as follows.

$$
\frac{\Delta I_{\text {point }}}{I_{0}}=\left[1-\exp \left(-h^{2} \Delta k^{2}\right)\right]^{\frac{1}{2}}
$$

Where $\Delta k$ is the change in wavenumber, $k=2 \pi / \lambda$. This equation can be applied equally to direct spectroscopy, in which case $\Delta \lambda$ denotes the width of the wavelength scan or of the region used to perform a baseline analysis or fit, or to WMS, in which case $\Delta \lambda$ denotes the wavelength modulation. For small $\Delta \lambda$, this approximates to the following, which is linear with $\Delta \lambda$ :

$$
\frac{\Delta I_{\text {point }}}{I_{0}} \approx 2 \pi h \frac{\Delta \lambda}{\lambda^{2}}
$$


where $\mathrm{h}$ is a characteristic of the diffuse object and represents the RMS path difference in reflected or transmitted light paths from the diffuser. For a simple, single scattering reflector, this would be equivalent to twice the mean surface height deviation. If we assume that $h \sim 6 \mu \mathrm{m}$, as for the experimental example given later in this paper, equation (7)'s deviation from linearity is less than $1 \%$ as long as $\Delta \lambda<14 \mathrm{~nm}$, therefore equation (8) can be used with confidence. Equations (7) and (8) can be considered improvement factors for the resulting speckle noise; integrating over $N$ independent speckles then yields the total RMS uncertainty in measured signal for small wavelength changes:

$$
\frac{\Delta I}{I_{0}}=\frac{2 \pi h}{\sqrt{N}} \frac{\Delta \lambda}{\lambda^{2}} .
$$

Here, $\Delta I$ represents the uncertainty in DC intensity in a direct spectroscopy experiment; the corresponding uncertainty in the $2^{\text {nd }}$ harmonic component in a WMS experiment can be found by reference to equation (3). The resulting measured uncertainty is given in Table 1 . The measured change in the speckle field therefore depends on the values of $\Delta \lambda$ and $h$. For $h \sim \lambda$ and $\Delta \lambda \sim 0.025 \mathrm{~nm}$, the level of uncertainty would be reduced by a factor of around $10^{4}$. Thus, there is a significant benefit to operating over a narrow spectral range - the narrower, the better. If one were to work with low pressure gases, with narrower linewidths, the uncertainty $\Delta \mathrm{I} / \mathrm{I}_{0}$ would reduce, as indeed it does in conventional cells. For large values of $h$, equation (7) tends to unity and there is no difference in the measured uncertainty between a speckle field under a large phase change and the uncertainty associated with independent static speckle fields.

For a well-developed (high contrast) speckle field, the ideal value of $h$ is considered to be of the order of the wavelength of light. As $h$ decreases from this value, the surface becomes more similar to a smooth optical surface with no benefit offered by scattering. At much higher values of $h$, a single scattering surface will behave like a connected series of smooth surfaces, exhibiting interference with a higher uncertainty in the integrated intensity. We have also used multiply scattering materials such as Spectralon $^{\mathrm{TM}}$, for which in reflective mode the light is believe to penetrate some distance (up to $10 \mathrm{~mm}$ ) before re-emerging as backscattered light. These materials produce well-developed, high contrast speckle fields, but the effective value of $h$ for such materials is unknown.

There is a final factor to consider, which is whether the speckle field moves during or between measurement integration periods (which in our experiments was defined by the time constant of our lockin amplifier). In the case of stable speckle, creating spectral features that do not change from one measurement to the next, subtraction of the zero baseline would reduce the speckle uncertainty to nil, though subsequent long-term drift of the speckle would re-establish the speckle related uncertainty. In the 
case of rapidly changing speckle, averaging several speckle fields during a measurement integration period reduces the statistical error. Goodman has shown that the addition of $M$ independent (uncorrelated) speckle fields reduces the speckle contrast by a factor of $\sqrt{ } M^{[22]}$, and we have confirmed this experimentally in a gas cell configuration, by statistical analysis of a single static speckle field ${ }^{[25]}$.

\subsection{Interferometric speckle}

In speckle interferometry, a speckle field is deliberately mixed with a reference beam ${ }^{[26]}$. In a gas cell, a reference beam might be formed by the Fresnel reflection from a smooth cell window, as shown in Figure 3.



Figure 3. Observation of interferometric speckle in an in-line configuration.

As the wavelength is scanned, or alternatively as the value of $z$ changes (for example because of thermal expansion), the resulting speckle patterns are correlated. By subtracting subsequent speckle images from one another (or by performing a baseline subtraction on a detected signal), interference features are revealed. In speckle interferometry these are termed correlation fringes and represent an intensity modulation of the randomised speckle field. We have previously demonstrated such an intensity modulation arising from a gas cell that uses a diffusely reflective surface ${ }^{[25]}$. Sirohi has shown that the intensity modulation at the detector takes the form ${ }^{[26]}$;

$$
I=I_{0}(1+A \cos \delta)
$$

where $\delta$ is the optical phase change induced by a change of wavelength or distance $z$. The constant $A$ is proportional to $\sqrt{I_{1} I_{2}}$, where $I_{1}$ and $I_{2}$ are the intensities of the reference beam and speckle field respectively. Thus these interference fringes are the direct analogy of etalon - induced fringes for conventional gas cells. 


\subsection{Self-mixing interference}

As an ideal diffuse surface scatters light in all directions, it can be hard to avoid a small proportion of this falling onto the laser diode. Figure 4 shows a simplified geometry.

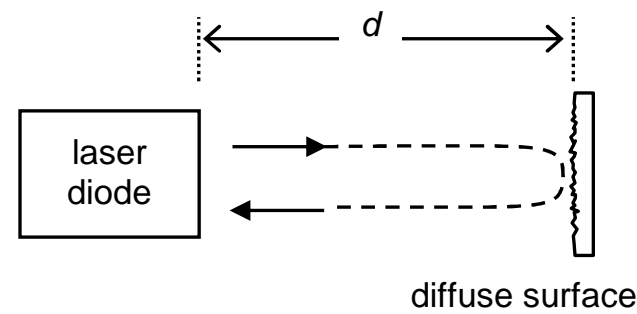

Figure 4. Formation of self-mixing interference.

Self-mixing or feedback interference results ${ }^{[27]}$, and the laser output intensity takes the form;

$$
I=I_{0}(1+m \cos \delta)
$$

where $\mathrm{m}$ is described as a modulation index (not to be confused with the modulation index used in TDLS described previously) and again $\delta$ is the optical phase change of the cavity. $\mathrm{m}$ is a function of the so-called feedback parameter $C$ (dependent on the characteristics of the laser) and, for very weak feedback, proportional to $\sqrt{r}$ where $r$ is the proportion of light reflected back into the laser diode. We have found that if steps are not taken to reduce feedback, the resulting interference fringes tend to dominate performance at low levels of $r^{[4]}$. However, as the interference is a modulation of the total laser emission, the effect is reduced to first order by the use of balanced detection schemes ${ }^{[4,28]}$.

It is worth noting that at higher levels of optical feedback, other laser instabilities can also result including the appearance of additional modes or, in extreme cases, coherence collapse. However, we have not experienced these problems in our work with optical diffusers.

\subsection{Summary}

Table 2 summarises the particular issues to be considered when using optical diffusers in TDLS. It is worth noting that the theory relating to laser speckle has, in general, been developed for use in the field of speckle interferometry and not rigorously tested in its application to TDLS. We assume here that random laser speckle may not be removed by baseline subtraction, therefore it is considered to define the fundamental performance-limiting uncertainty of the systems we have studied. The other problems 
highlighted may be reduced by good design, at least in principle. Speckle uncertainty may be reduced by the use of a high NA lens and / or a large detector. The use of diffuse optics generally results in a loss of optical throughput because light is scattered in all directions and not collected by the detector. Fortunately, use of a high NA lens is also the logical response to such conditions. In a collimated system, use of an additional transmissive lens might introduce interference fringes, however in this case the light is uncollimated. We have not studied whether this contributes to a lower level of interference for reflections within the lens or between the lens and the detector. If such interference were troublesome, an off-axis reflective lens might be used instead.

Table 2. Summary of issues to be considered in TDLS gas cells using optical diffusers.

\begin{tabular}{|l|l|c|}
\hline$\underline{\text { Issue }}$ & Reduction strategy & $\underline{\text { Resultant level, as } \Delta I / I_{0}}$ \\
\hline Random laser speckle & $\begin{array}{l}\text { Use large apertures } \\
\text { Move the sample } \\
\text { Use wavelength } \\
\text { modulation or baseline } \\
\text { reduction }\end{array}$ & $\begin{array}{c}\text { Fundamental uncertainty in } \\
\text { the range } \\
2 \times 10^{-3}-2 \times 10^{-7}\end{array}$ \\
\hline Interferometric speckle & $\begin{array}{l}\text { AR coat and misalign cell } \\
\text { window }\end{array}$ & $\begin{array}{l}\text { Analogous to conventional } \\
\text { etalon fringes } \\
\leq-6\end{array}$ \\
\hline Self-mixing interference & $\begin{array}{l}\text { Good quality isolation } \\
\text { Balanced detection }\end{array}$ & $\leq 10^{-5}-10^{-7}$ \\
\hline
\end{tabular}

In this work, we chose to use WMS with $2^{\text {nd }}$ harmonic demodulation. For our experimental purposes, compared to direct spectroscopy, this gave us the advantages of a zero baseline, a rapid response using our equipment and no dependence on spectral fitting algorithms. However, as the purpose of this paper is to investigate laser speckle in cells using optical diffusers, the principles should be capable of being translated to other spectroscopic techniques.

\section{Gas cell design}

We consider first a simple reflective gas cell and calculate the expected uncertainty in the measurement using the above theory. Figure 5 shows a schematic of the proposed cell geometry. Light from a laser 
diode passes through a beamsplitter, enters the cell via a wedged and AR coated window and strikes a diffuse reflector after making a pass though the cell. A small proportion of the light is backscattered along the incident axis and makes a second pass through the cell before being deflected by the beamsplitter towards a photodiode.

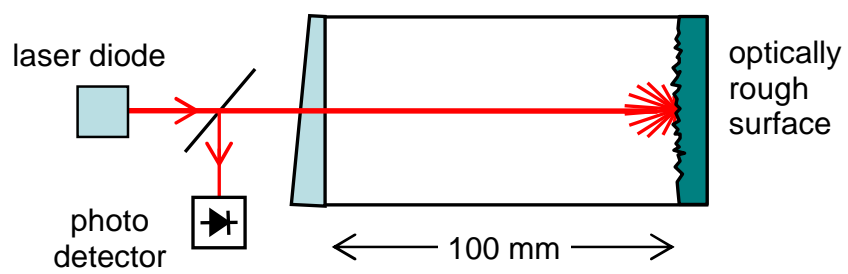

Figure 5. Schematic diagram of a gas cell using a diffuse optical reflector.

We estimate that the minimum practical value of $\mathrm{z}$ for this arrangement would be $150 \mathrm{~mm}$, which for our other constraints $(d=25 \mathrm{~mm}, D=1 \mathrm{~mm}, \lambda=1651 \mathrm{~nm})$ yields a proportional uncertainty of $\Delta I / I_{0}=10^{-2}$. This could be improved under wavelength modulation by up to $\Delta I / I_{0}=10^{-6}$ for an optimum value of $h$ and $\Delta \lambda=0.025 \mathrm{~nm}$. The cell has the advantage of using a double pass to improve detection limits. However, a number of potential disadvantages are also present. Firstly, there is a possibility of generating interferometric speckle with this arrangement. Although this would be avoided by misaligning a single window, the window is relatively close to the detector and the degree of misalignment required would be consequently greater. Secondly, the configuration has a relatively poor throughput as there is no lens to improve the collection efficiency at the detector, and the use of a beamsplitter exacerbates the problem. Finally, stray reflections associated with plate or cube beamsplitters may cause additional interference effects. Although pellicle beamsplitters avoid these problems, they are fragile and relatively expensive.

The same principles were applied to a range of different gas cell design and the results are compared in Table 3. We have also compared the typical performance of a cell with a conventional configuration. In all cases we have used the same constraints, however depending on the geometry this might result in slightly different values of $z$, as in the case above. It was decided to implement the final transmissive design in our experiments for a number of reasons. The design is simple, and offers more convenient reduction of both interferometric speckle and self-mixing interference, the latter because the majority of the light is scattered in the forward direction, away from the laser diode. 
Table 3: Summary of gas cell designs.

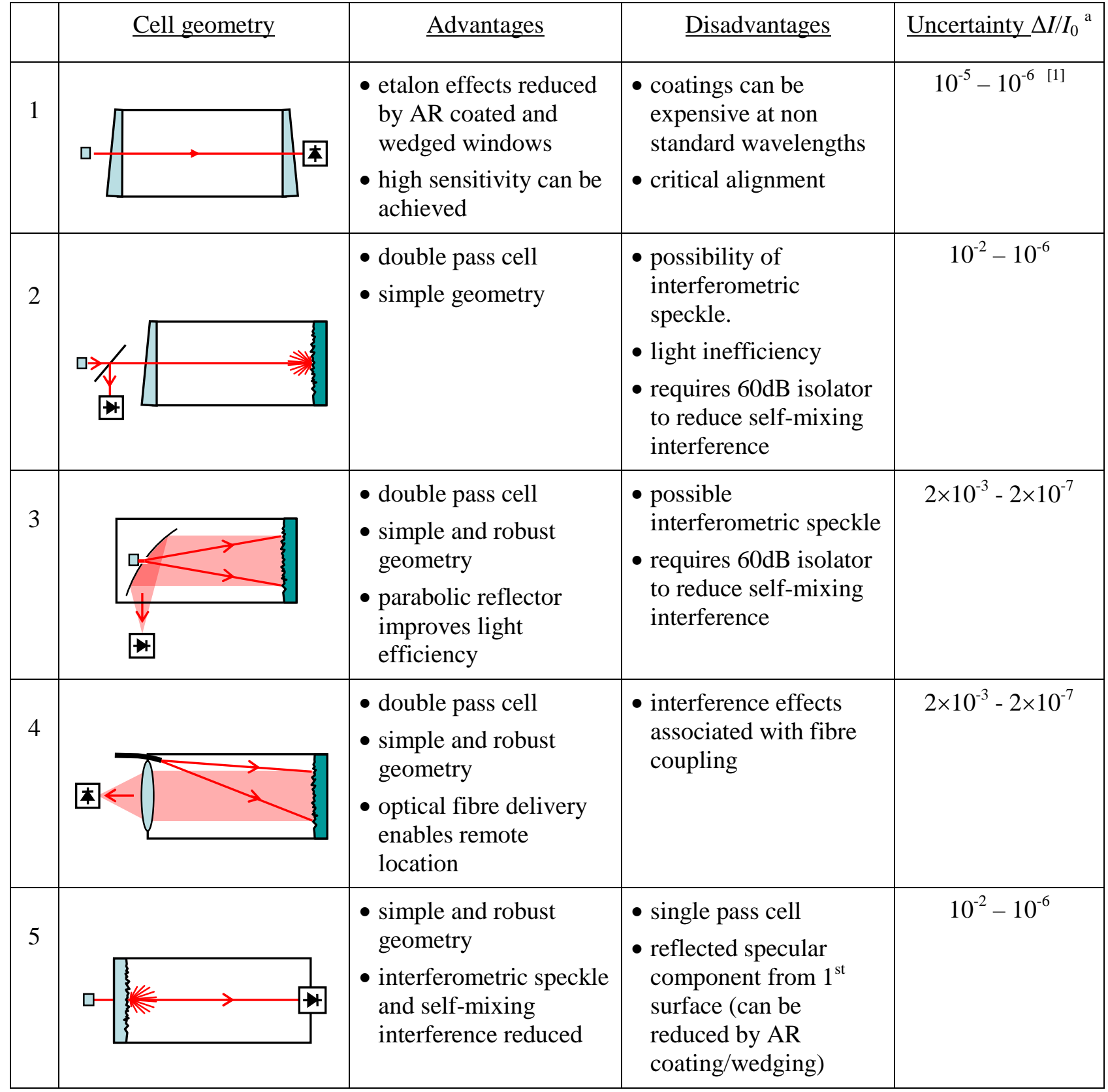

a $100 \mathrm{~mm}$ cell length, $\max 25 \mathrm{~mm}$ diameter optics, $\lambda=1651 \mathrm{~nm}, D=1 \mathrm{~mm}, \Delta \lambda=0.025 \mathrm{~nm}, h \geq \lambda$

In all the gas cell designs considered here, the estimated speckle related intensity uncertainty for a single static speckle pattern remained in the range $10^{-2}$ to $2 \times 10^{-3}$. This may be improved by increasing the detector size; a $5 \mathrm{~mm}$ diameter detector would give a 5-fold improvement in the signal : noise ratio. However, these levels are not sufficient to enable ppm level gas detection. Making the measurement over 
a narrow spectral range $(\Delta \lambda= \pm 0.025 \mathrm{~nm}$ for WMS with methane at $1651 \mathrm{~nm})$ would improve the signal : noise ratio by up to a factor of $10^{4}$, depending on the surface properties.

To achieve this potential, it is therefore important that the diffuse surface is chosen carefully, and that the speckle field does not move substantially between, for example, intensity measurements taken at each side of the absorption line. The same issue exists for measurements in conventional cells; where vibrations are present, modulation frequencies should ideally be greater than the frequency of mechanical vibration so that the baseline remains stable during a single scan or wavelength modulation cycle.

Objective speckle uncertainty can be reduced by the use of a large detector and large illuminated area. When a lens is used to collect light (subjective speckle), the level of associated speckle uncertainty can be reduced using a high NA lens, which also improves the light collection efficiency. The speckle size is then independent of illumination geometry, which has advantages for measurements made in locations where the backscattering surface is difficult to control (for example, using backscatter laser pointers).

\section{Experimental details}

We employed WMS at $\mathrm{f}=6 \mathrm{kHz}$ with second harmonic (2f) detection at $12 \mathrm{kHz}$. Figure 6 shows a schematic diagram of our laser diode modulation and detection apparatus, in this case configured for use with our transmissive gas cell design. A sinusoidal signal at $6 \mathrm{kHz}$ from a signal generator (Hewlett Packard HP33120A) was applied to the laser controller (ILX Lightwave, ILX LDC-3722B) to give an amplitude at the laser diode of $24 \mathrm{~mA}$ (peak to peak). Our DFB laser diode package (Semelab Ltd) incorporated a $1651 \mathrm{~nm}$ laser (NEL NLK1U5C1CA-TS) collimated with an aspheric lens (Lightpath 350230D). Gross wavelength tuning was achieved by controlling the diode temperature using a Peltier element within the package. 




Figure 6. Schematic experimental configuration for $2 \mathrm{f}$ wavelength modulation spectroscopy, using a $3 \mathrm{f}$ line lock.

A proportion of the laser beam ( $8 \%$ ) was sampled by a pellicle beamsplitter (Thorlabs BP208) to a gas reference cell (PD3 in Figure 6) consisting of a Ge detector at the bottom of a TO18 can containing 100\% methane, giving an effective pathlength of $5.3 \mathrm{~mm}$. We would wish to avoid the use of such beamsplitters in field applications, for example by using a partial reflection from a window or from the diffuser, or by using a proportion of the transmitted beam. However, for these experiments the use of beamsplitters permitted simpler comparison of different cell geometries. The signal from the photodiode was demodulated at 3f using a lock-in amplifier (Stanford SR850) to provide an error signal which was fed, via a PID (proportional, integrative, derivative) controller to the laser controller, controlling the dc current of the laser diode so as to lock the emission wavelength to the gas absorption line centre. In our standard WMS experiments, the laser emission was thus actively locked and we did not perform a simultaneous wavelength scan. However, in some experiments the lock was not used and a simultaneous slow wavelength scan through the gas absorption line was performed in order to observe the $2 \mathrm{f}$-demodulated spectrum.

The laser beam striking the optical diffuser had a diameter of approximately $8 \mathrm{~mm}$. A proportion of the forward scattered light fell onto the detector / amplifier (PD1: Thorlabs, PDA50EC, 1mm diameter) after making a single pass across the cell. A lock-in amplifier (Stanford Research Systems SR850, time constant $\tau=1 \mathrm{~s}$ ) was used for $2 \mathrm{f}$ demodulation, its output voltage (X, in $\mathrm{X}, \mathrm{Y}$ mode) sampled using a data 
acquisition card and transferred to a computer running Labview ${ }^{\mathrm{TM}}$ software, through a data acquisition card (National Instruments PCI 6259). The DC signal from PD1 was sampled using a digital multimeter (DMM, Keithley 195A) before also being passed to the PC for data processing. Monitoring the DC level allowed us to normalise the $2 \mathrm{f}$-demodulated signals to compare results between different optical diffusers, each of which could project a different level of light onto PD1. A second detector amplifier (PD2 in Figure 6) was used to measure a proportion (approximately 45\%) of the incident beam intensity via a beamsplitter (Thorlabs BP245B3). This reference detector channel employed the same model of detector / amplifier, lock-in amplifier and DMM, with the same set-ups, as the main signal channel from PD1. Both the sample cell and the reference detector PD2 were angled slightly (around 10 ${ }^{\circ}$ with respect to the optical path.

Test gases were fed to the gas cell from certified cylinders (Scott Specialty Gases), one containing hydrocarbon (HC) free air and the other containing either $1010 \mathrm{ppm}$ or $50.1 \mathrm{ppm}$ methane in $\mathrm{HC}$ free air. A bank of mass flow controllers (Teledyne Hastings HFC-302 with THPS-400 controller) was used to control flow rates from the two cylinders, with downstream mixing generating different concentrations in the range $0-1010 \mathrm{ppm}$.

\section{$5 \quad$ Results}

We tested the chosen geometry of section 3, namely cell 5: a simple transmission cell design in which light from the laser diode passed through an optical diffusive element, which scattered the light mainly in the forward direction. A proportion of the light fell onto a $1 \mathrm{~mm}$ diameter photodiode after making a single pass across a 100mm gas cell. For the purpose of comparison, we also tested a conventional transmission cell of the same length, employing two wedged and anti-reflection coated windows.

\subsection{Choice of materials}

Different types of diffusely transmitting materials were investigated. Their relative levels of transmission were compared for a $1 \mathrm{~mm}$ detector placed at a distance of $100 \mathrm{~mm}$, and we observed the form of the scattered light pattern that they produced. The results are shown in Table 4. 
Table 4. Transmissive diffusers tested and their optical properties

\begin{tabular}{|l|l|c|l|}
\hline Diffuser material & Characteristics & Relative transmission & Light pattern \\
\hline No diffuser & open space & $100 \%$ & collimated beam \\
\hline $\begin{array}{l}\text { Ground BK7 glass } \\
\text { (Thorlabs DG10) }\end{array}$ & 220 grit & $21 \%$ & strong specular reflection \\
\cline { 2 - 4 } & 600 grit & $58 \%$ & well-developed speckle \\
\cline { 2 - 4 } & 1500 grit & $48 \%$ & $\begin{array}{l}\text { part of beam not diffusely scattered, } \\
\text { but displaced by angling surface }\end{array}$ \\
\hline $\begin{array}{l}\text { Zenith } \\
\text { (Sphere Optics DF- } \\
\text { 100) }\end{array}$ & $100 \mu \mathrm{m}$ thick & $4.0 \%$ & $\begin{array}{l}\text { well-developed speckle, best used } \\
\text { in reflection }\end{array}$ \\
\cline { 2 - 4 } $\begin{array}{l}\text { Depolariser } \\
\text { (Thorlabs DPU-25- } \\
\text { C) }\end{array}$ & AR coated & $2.2 \%$ & $\begin{array}{l}\text { collimated beam, interference } \\
\text { fringes }\end{array}$ \\
\hline $\begin{array}{l}\text { Holographic diffuser } \\
(\text { Luminit }\end{array}$ & $0.5^{\circ}$ circle pattern & $>70 \%$ & $\begin{array}{l}\text { Scattered light over specified cone } \\
\text { angle }\end{array}$ \\
\hline
\end{tabular}

We chose to use a 1500 grit ground glass diffuser because it combined a well-developed speckle pattern, good throughput and minimal specular reflection. The mean surface height deviation is uncertain but expected to be at or below $12.6 \mu \mathrm{m}$, as the ISO specification for 1500 grit refers to abrasion by particles with a mean diameter of $12.6 \mu \mathrm{m}$. For a material of refractive index 1.5 , this gives a mean optical pathlength difference $h<6 \mathrm{~m}$ for a transmitter, and we would expect the improvement in noise level calculated using equation (8) to be less than or equal to a factor of $2.8 \times 10^{4}$.

\subsection{Gas absorption measurements with a diffuse cell}

The transmission cell was built using a 1500 grit ground glass window (Thorlabs DG10-1500) and 100mm length, with a detector / amplifier (Thorlabs, PDA50EC) integrated into the end so as to avoid the use of additional windows. The only factor to be considered during alignment was to misalign the Fresnel reflection from the front (smooth) surface of the diffuser plate, therefore the process was very quick ( $<1 \mathrm{~min})$. A separation of over 1 metre between the laser diode and the cell ensured that self-mixing interference effects were minimised. The output was recorded for $2^{\text {nd }}$ harmonic WMS with a $3 f$ line lock while a series of gas mixtures of different concentrations was passed through the cell. For each concentration point, zero data were recorded before and after (over a total time period of approximately 6 minutes) in order to remove any effects of drift. Figure 7 shows the results; a short-term noise equivalent absorbance (NEA) of $2 \times 10^{-5}$ is estimated, corresponding to around $5 \mathrm{ppm}$ methane $(1 \sigma)$. 


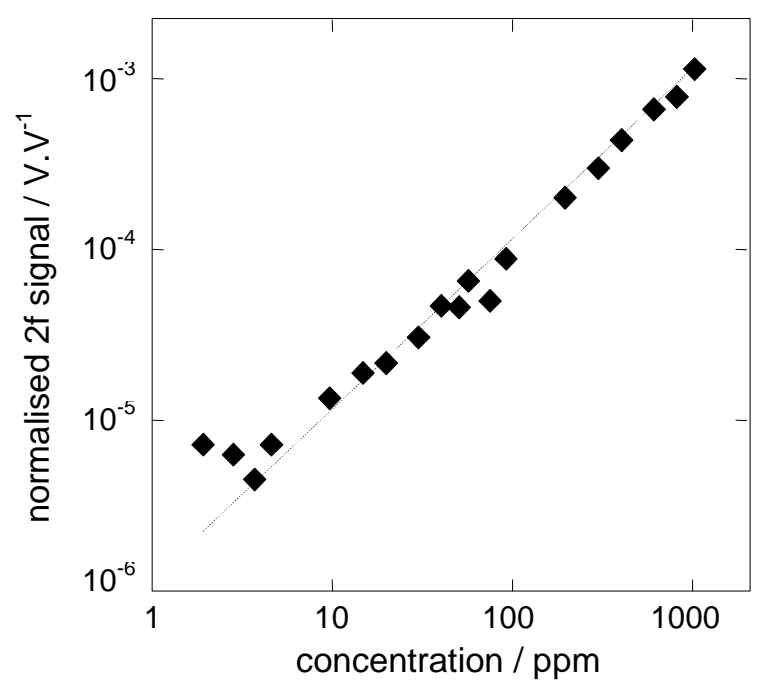

Figure 7. $2 \mathrm{f}$ - demodulated signals versus gas concentration for cell using a transmissive optical diffuser. $2 \mathrm{f}$ signals have been normalised by dividing by the measured DC signal, and the dashed line shows a linear response.

\subsection{Assessment of drift}

The level of system drift was assessed by continuously recording data (with no gas in the cell) over a 22 hour period, for both the signal channel PD1 and the reference channel PD2. Allan variance plots $\left(\sigma^{2}\right.$ as defined by Werle et al. ${ }^{[29]}$ ) were calculated for the recorded $2 \mathrm{f}$ measurements for each channel, using data normalised by division by the DC signals. These plots are shown in Figure 8 and describe a system dominated by drift. Despite the use of normalised data for both channels, the Allan variance seems to be a factor of 3-10 times more pronounced on the reference channel than the signal channel. The drift on the signal seems to be bounded at a mean value $\left(\sqrt{ }\left(\sigma^{2}\right)\right)$ of an NEA of $3 \times 10^{-4}$, and at a value of $7 \times 10^{-4}$ on the reference channel. Because of the high level of drift on the reference channel, we cannot associate the drift on the signal channel with the use of the diffuse optical cell alone, rather this data puts an upper limit on what the effect of the cell might be. The worst measured value of $\sqrt{ }\left(\sigma^{2}\right)$ corresponds to a concentration of around 70 ppm, using Figure 7. 


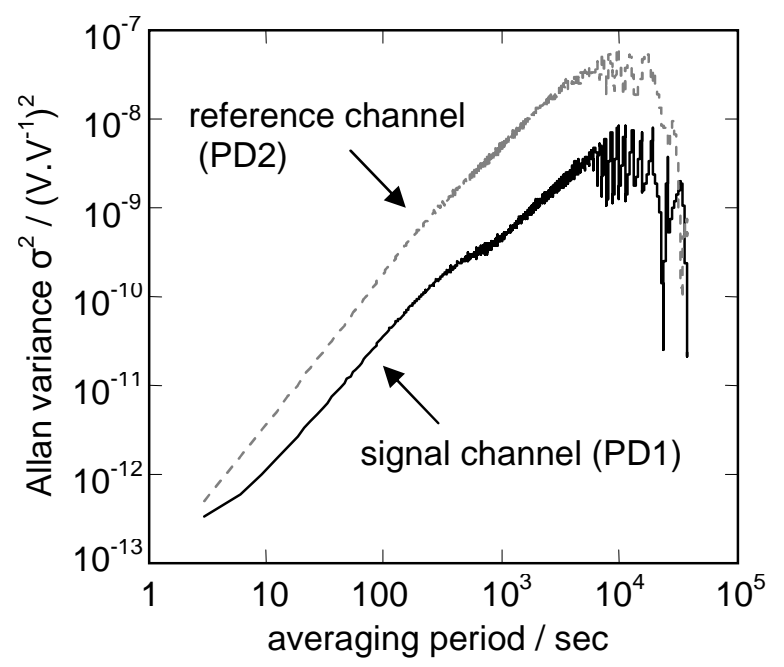

Figure 8. Allan variance plot for normalised $2 \mathrm{f}$ time series data taken over a 22 hour period with an empty cell.

The data is consistent with an interference effect; interference fringes have finite extent and would limit drift to a maximum value. We are aware of interference fringes in our laser diode output with a wide FSR, which cause instabilities similar to those seen on the reference channel. It is curious however that the Allan variance for the signal channel PD1 was consistently lower than that for the reference channel PD2, despite both these measurements being normalised (2f measurement / DC measurement) and using identical apparatus. We were hoping to use the reference channel to correct for instabilities in the laser diode output, but this was not possible as the outputs from PD1 and PD2 were uncorrelated. Further work is needed to investigate the source of the drift in our system. Two additional effects may be present; (i) our pellicle beamsplitter may suffer from low frequency movement, which would affect the reflected reference beam more than the transmitted signal beam, and (ii) the signal channel may be measuring over a wider and more representative sample of the emitted laser beam than our reference channel.

\subsection{Summary of results with transmissive cell}

We consider speckle uncertainty on the detector to determine the fundamental limit of detection of this cell design. It would be improved however by the use of a more divergent illumination of the ground glass plate and / or the use of a high NA lens to collect light onto the photodiode. In our experiments, we have established a short-term ( 6 minute) limit of detection of around 5 ppm, in a system dominated by drift. 
For a spectral scan of $\Delta \lambda=0.025 \mathrm{~nm}$, beam diameter $d=8 \mathrm{~mm}$, distance to detector $z=100 \mathrm{~mm}$, detector diameter $D=1 \mathrm{~mm}$ and surface roughness deviation $h \leq 6 \mu \mathrm{m}$, we would expect the level of speckle uncertainty to be $\Delta I / I_{0} \sim 10^{-5}$, corresponding to an NEA for WMS of around $1.5 \times 10^{-5}$. Using equation (3), for a $100 \mathrm{~mm}$ pathlength cell and absorptivity of $0.38 \mathrm{~cm}^{-1}$ at the methane line centre, this translates to a detection limit of $4 \mathrm{ppm}$ or below. Although this is consistent with our short-term results for gas concentration measurement (an NEA of $2 \times 10^{-5}$ ), it is not known to what extent the spectral effect of speckle was stable during these experiments.

The drift experiment puts an upper limit on the degree of instability of the speckle, and thereby the speckle uncertainty at an NEA of $3 \times 10^{-4}$. Unfortunately, our experimental platform was not itself stable and its performance may have dominated these results. Work is in progress to identify and remove the remaining causes of instability, which could be associated with feedback effects within the laser diode package ${ }^{[4]}$, or by movement of our pellicle beamsplitter. The level of speckle uncertainty calculated in the absence of wavelength modulation (equation (6), Table 1 column 2) does not apply.

\subsection{Speckle noise reduction}

Two methods have been tested to suppress speckle noise by rotating or vibrating the diffuser. In these experiments, the speckle related intensity uncertainty was deliberately exaggerated, by using a small detection aperture, so that the effectiveness of the suppression could be quantified unambiguously. For simplicity the moving diffuser was placed outside a conventional gas cell, as shown in Figure 9. In both cases, a $1 \mathrm{~mm}$ thick sample of Zenith ${ }^{\mathrm{TM}}$ (proprietary PTFE, Sphere Optics) was used as a reflective diffuser. We minimised the level of backscattered light returned to the laser diode by using a large angle of incidence and by placing the laser diode at a distance of over $1 \mathrm{~m}$ from the diffuser. 

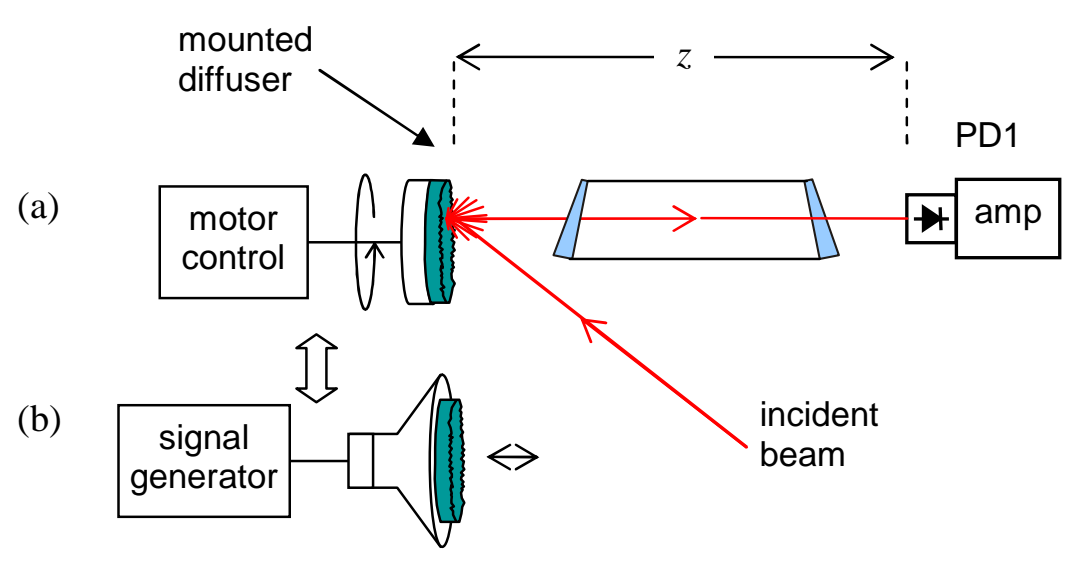

Figure 9. Experimental configuration for speckle noise suppression using moving diffuse reflectors, by (a) rotation of a motor, and (b) vibration of a loudspeaker.

The speckle size was exaggerated by increasing the distance $z$ between the diffuser and the detector to around $600 \mathrm{~mm}$. The laser drive current was ramped over the range $35-70 \mathrm{~mA}$ in steps of $0.2 \mathrm{~mA}$, while $2 \mathrm{f}-$ demodulated detector output was recorded. In the first experiment we compared a stationary and rotating diffuser, the latter consisting of a $36 \mathrm{~mm}$ diameter, $1 \mathrm{~mm}$ thick disc of Spectralon ${ }^{\mathrm{TM}}$ rotated at a frequency of $1 \mathrm{kHz}$ using the motor from an optical chopper. A lock-in time constant of $\tau=100 \mathrm{~ms}$ was used to allow scans to be completed within a reasonable time period. Scans were taken with the cell evacuated and also with the cell filled with $1.25 \%$ methane at atmospheric pressure. The resulting $2 \mathrm{f}$-demodulated gas signals are illustrated in Figure 10 and show a large reduction in the received noise level when the diffuser was rotating. 


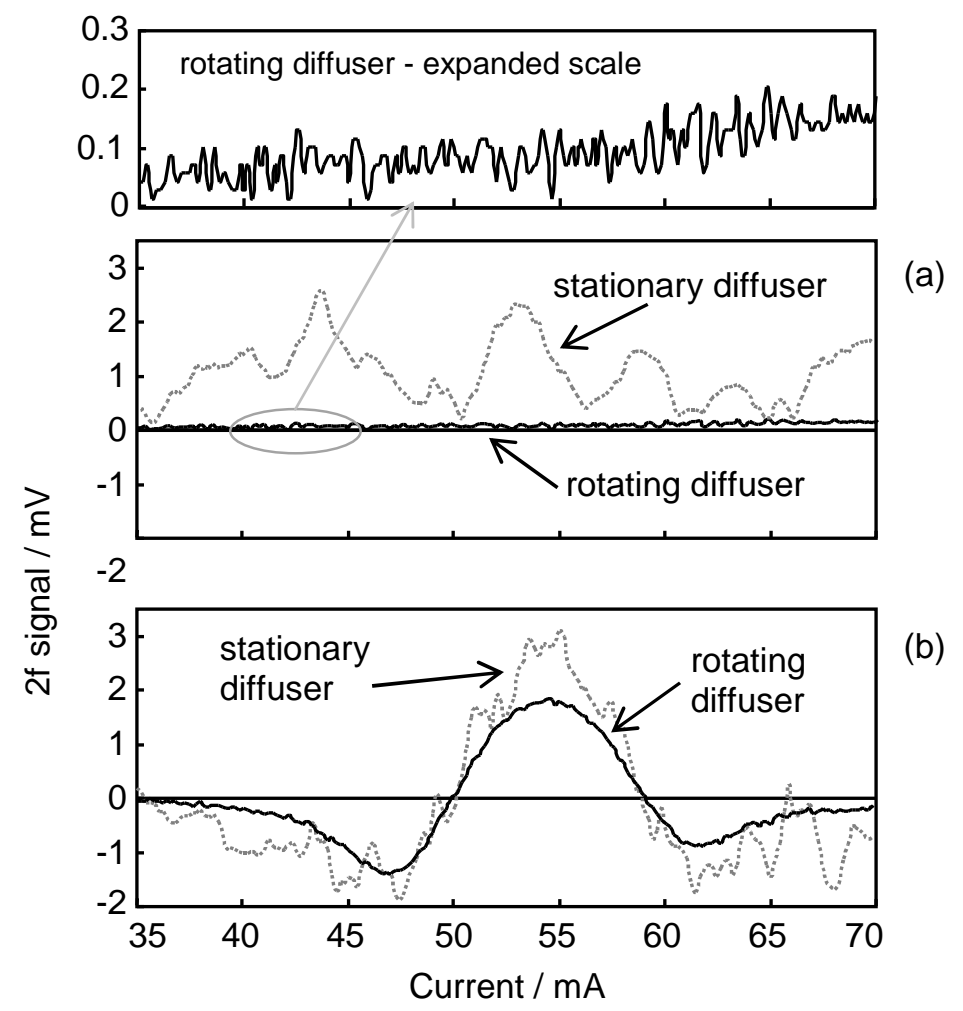

Figure 10. Speckle noise reduction achieved by rotating a diffuser. 2f-demodulated WMS scans of (a) HC free air, and (b) methane at a concentration of $1.25 \%$ vol. An exaggerated level of speckle noise was used to demonstrate the effect.

Within one measurement integration period of $1 \mathrm{~s}$, we expect to have averaged over the entire rotation of the diffuser, which for an $8 \mathrm{~mm}$ diameter beam striking the diffuser approximately $10 \mathrm{~mm}$ from the centre of rotation gives an equivalent of 8 independent speckle fields, which we would expect to yield an improvement in the noise level of $\sqrt{ } 8=2.8$. A quantitative analysis of the results in Figure 10 yields an improvement factor of 13 , which is much greater. It is possible that additional speckle averaging was provided by imperfect alignment and diffuser wobble, giving a larger number of independent speckle patterns and a greater degree of suppression.

In a second experiment, we glued the diffuser (36mm diameter) to a loudspeaker diaphragm (Goldstar

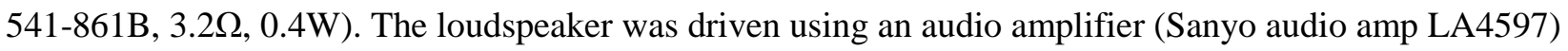
with a $200 \mathrm{~Hz}$ sine wave, the amplitude adjusted manually using the amplifier's volume control (gain) to minimise ripple on the detector output. Vibrating the diffuser resulted in an observable reduction in speckle contrast, however the resulting amplitude of vibration is unknown. The results of a $2 \mathrm{f}$ 
demodulated methane line scan are shown in Figure 11. Quantitative analysis shows that the speckle noise was reduced by a factor of 10 in this case.
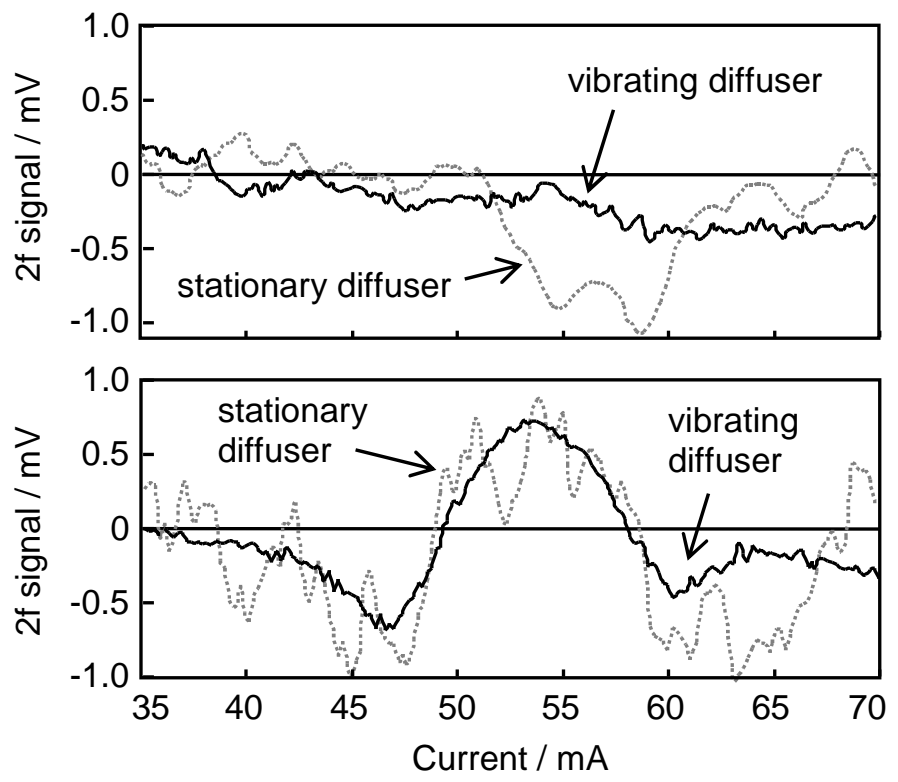

Figure 11. Speckle noise reduction achieved by rotating a diffuser. 2f-demodulated WMS scans of (a) HC free air, and (b) methane at a concentration of $1.25 \%$ vol. An exaggerated level of speckle noise was used to demonstrate the effect.

\section{Discussion and conclusions}

We have analysed a number of factors affecting cell performance when optical diffusers are used instead of conventional, smooth windows or mirrors. Self-mixing or feedback interference can be performance limiting for both conventional and diffuse cells, but can be difficult to avoid in the latter, especially when diffuse reflectors are used. Our laser diode, which employed a relatively large (5 mm diameter) collimating lens for the purpose of our experiments, was particularly susceptible to this interference effect. To ensure that it did not affect our measurements, we used a large separation between the laser diode and the cell, but a more practical solution would be needed for field instruments. This could include a smaller laser diode aperture, better optimised cell design and the use of an isolator.

Interferometric speckle in diffuse gas cells is the direct analogue of etalon-induced interference fringes in conventional cells and therefore needs to be removed. We have found in practice that it has not presented a problem and that its removal is simpler than for conventional cells, since the number of specular 
reflections from optical surfaces is reduced. Our chosen diffuse cell, with a transmission design, also minimises the possibility of generating a specular reference beam that can interfere with the speckle field.

Random laser speckle is believed to be a fundamental, performance-limiting effect for the diffuse optics that we have used. To gain the benefits of optical diffusers requires surface roughness that is comparable with the illumination wavelength, therefore speckle fields will be well-developed with high contrast. We have calculated the resulting speckle uncertainty for absolute measurements of light transmission, and for self-referenced measurements taken over spectral scans with a small $\Delta \lambda$, by baseline subtraction or using WMS. According to established speckle theory, the latter should offer levels of uncertainty compatible with ppm level gas detection, with $\Delta I / I_{0}$ in the range $10^{-2}-10^{-6}$ for our chosen $100 \mathrm{~mm}$ cell. The precise degree of uncertainty is dependent on the equivalent surface roughness of the optical diffuser. While this may be determined for simple, singly scattering materials such as the ground glass used in our chosen design, its value is unknown for multiply scattering bulk reflectors such as Spectralon ${ }^{\mathrm{TM}}$ and Zenith ${ }^{\mathrm{TM}}$ PTFE. It is also possible that multiple scattering in such materials will reduce uncertainty further through depolarisation $^{[25]}$.

Cells employing optical diffusers are simpler to align than standard cells. With careful design, the level of uncertainty determined by speckle on the detector was estimated for our system to give an NEA for WMS of around $1.5 \times 10^{-5}$. Our short-term experimental results are consistent with this, showing a short-term NEA $(1 \sigma)$ of $2 \times 10^{-5}$, but longer term drift of up to $3 \times 10^{-4}$ over several hours. There may be additional sources of drift in our system that require further attention. We have also demonstrated two methods to reduce speckle uncertainty by a factor of 10 or more, based on vibration or rotation of the diffuser. These are analogous to the use of dithered optical window or spoiler plates in conventional cells, to reduce the visibility of interference fringes.

\section{Acknowledgements}

This work was carried out under an EPSRC research grant (GR/T04601/01). Jane Hodgkinson is supported by an EPSRC Advanced Research Fellowship (GR/T04595/01). We would like to thank Dan Francis for his help with our data acquisition software.

\section{References}

[1] H. I. Schiff, G. I. Mackay, J. Bechara, Chapter 5 in Air Monitoring by Spectroscopic Techniques, M 
W Sigrist, Ed, M Wiley, New York (1994)

[2] J. A. Silver, A. C. Stanton, Appl. Opt. 27 (10), 1914-1916 (1988).

[3] J. Reid, J. Shewchun, B. K. Garside, E. A. Ballik, Appl. Opt. 17 (2), 300-307 (1978).

[4] D. Masiyano, J. Hodgkinson, S. Schilt, R. P. Tatam, Appl. Phys. B 96 (4), 863 (2009).

[5] P. Werle, Spectrochim. Acta A 54 (2), 197-236 (1998).

[6] D. S. Bomse, A. C. Stanton, J. A. Silver, Appl. Opt. 31 (6), 718-30, 1992.

[7] J Reid, M El-Sherbiny, B K Garside, E A Ballik, Appl. Opt. 19 (19), 3349-3354 (1980).

[8] C. B. Carlisle, D. E. Cooper, H. Prier. Appl. Opt. 28 (13), 2567-2576 (1989).

[9] F. Capellani, G. Mellandrone, G. Restelli, In Monitoring of Gaseous Pollutants by Tunable Diode Lasers, ed. R. Grisar, H. Preier, G. Schmidtke, G. Restelli (UK: Kluwer Academic Publishers) pp 5160 (1987).

[10] S. Chou, D. S. Baer, R. K. Hanson, Appl. Opt. 36 (15), 3288-3293 (1997).

[11] C. R. Webster, J. Opt. Soc. Am. B 2 (9), 1464-1470 (1985).

[12] J. Hodgkinson, R. D. Pride, C. Tandy, D. G. Moodie, G. Stewart, Proc SPIE 4074, 90-98, Applications of Optical Fiber Sensors, Alan J. Rogers; Ed (2000).

[13] J. Chen, A.Hangauer, R. Strzoda, M.-C. Amann, Laser spectroscopic oxygen sensor using diffuse reflector based optical cell and advanced signal processing, accepted for publication, Appl. Phys. B, this issue.

[14] T. Svensson, M. Andersson, L. Rippe, S. Svanberg, S. Andersson - Engels, J. Johansson, S. Folestad, Appl. Phys. B 90, 345-354 (2008).

[15] M. Lewander, Z. Guan, K. Svanberg, S. Svanberg, T. Svensson, Opt. Express 17 (13), 10849- 10863 (2009).

[16] T. Iseki; H. Tai, K. Kimura, Meas. Sci. Technol. 11, 594-602 (2000).

[17] R.T. Wainner, B.D. Green, M.G. Allen, M.A. White, J. Stafford-Evans, R. Naper, Appl. Phys. B 75, 249-254 (2002).

[18] B. van Well, S. Murray, J. Hodgkinson, R. Pride, R. Strzoda, G. Gibson, M. Padgett, J. Opt. A 7, S420-S424, (2005).

[19] L. S. Rothman et al, J. Quant. Spectrosc. Ra. 96, 139-204 (2005).

[20] W. Jin, Y. Z. Xu,., M. S. Demokan, G. Stewart. Appl. Opt. 36 (28), 7239-7246 (1997).

[21] J. A. Silver, Appl. Opt. 31 (6), 707-717 (1992).

[22] J. W. Goodman, Chapter 2 in Laser Speckle and Related Phenomena, ed JC Dainty, Springer Verlag (1975).

[23] A. E. Ennos, Chapter 6 in Laser Speckle and Related Phenomena, J C Dainty, Ed, Springer-Verlag, 
(1975)

[24] H. Fujii, J. M. Y. Lit, Opt. Commun. 22 (2), 231-235 (1977).

[25] D. Masiyano, J. Hodgkinson, R. P. Tatam, Appl. Phys. B 90, 279-288 (2008).

[26] R. S. Sirohi, Contemp. Phys. 43 (3), 161-180 (2002)

[27] G Giuliani, M Norgia, S Donati, T Bosch, J. Opt. A: Pure Appl. Opt. 4, S283-294 (2002)

[28] L. Persson, F. Andersson, M. Andersson, S. Svanberg. Appl. Phys. B 87, 523-530, 2007.

[29] P. Werle, R. Mücke, F. Slemr. Appl.Phys. B 57, 131-139, 1993. 